\title{
Alterações morfológicas placentárias de recém-nascidos pequenos para a idade gestacional
}

\author{
Changes in placental morphology of small for gestational age newborns
}

\author{
Lúcio H. Oliveira ${ }^{1}$, César C. Xavier ${ }^{2}$, Ana M.A. Lana ${ }^{3}$
}

\section{Resumo}

Objetivo: verificar a morfologia placentária de recém-nascidos a termo pequenos para a idade gestacional, tendo como hipótese a existência mais freqüente de alterações placentárias em recémnascidos pequenos para a idade gestacional do que em adequados para a idade gestacional.

Métodos: realizou-se estudo transversal, na maternidade Terezinha de Jesus, em Juiz de Fora, MG, no período compreendido entre fevereiro e novembro de 1996, no qual foram coletados dados referentes a cinqüenta recém-nascidos a termo, estimados como pequenos para a idade gestacional. Como grupo controle, foram incluídos recém-nascidos a termo, estimados como adequados para a idade gestacional, randomizados na proporção de um controle para cada caso. Dos 100 recém-nascidos participantes do estudo, foram obtidas as placentas, cordão umbilical e membranas, que foram examinados no Laboratório de Histologia e Embriologia do Departamento de Morfologia da UFJF e no Departamento de Anatomia Patológica e Medicina Legal da UFMG. As mães foram entrevistadas, e os recém-nascidos avaliados quanto à idade gestacional, peso, comprimento e perímetro cefálico.

Resultados: as placentas dos recém-nascidos pequenos para a idade gestacional apresentaram maior incidência de corioamnionite, infarto placentário, deposição perivilosa extensa de fibrina e vilosite crônica em focos múltiplos de localização parabasal, além de mostrarem menor peso e menores diâmetros em relação às placentas do grupo de recém-nascidos adequados para a idade gestacional $(\mathrm{p}<$ $0,05)$. O índice placentário mostrou-se significativamente maior no grupo pequenos para a idade gestacional $(\mathrm{p}<0,05)$, indicando que o comprometimento do peso fetal foi relativamente maior que o comprometimento de peso da placenta.

Conclusão: as placentas dos recém-nascidos pequenos para a idade gestacional apresentam diferenças anatomopatológicas e morfométricas, estatisticamente significativas, em relação aos recémnascidos adequados para a idade gestacional.

J Pediatr (Rio J) 2002; 78 (5): 397-402: recém-nascido pequeno para a idade gestacional, retardo do crescimento intra-uterino, placenta.

1. Prof. Assistente da Universidade Federal de Juiz de Fora (UFJF). Mestre em Pediatria pela Faculdade de Medicina da Universidade Federal de Minas Gerais (FM-UFMG), Belo Horizonte, MG.

2. Prof. Adjunto, Doutor em Pediatria, FM-UFMG.

3. Prof. Adjunto, Doutora em Anatomia Patológica, FM-UFMG.

Artigo submetido em 04.07.01, aceito em 19.06.02.

\begin{abstract}
Objective: to verify changes in placental morphology of small for gestational age newborns, considering that the occurrence of placental alterations is more frequent in small for gestational age (SGA) infants than in appropriate for gestational age (AGA) infants.

Methods: fifty SGA newborns were included in a cross-sectional study, which involved gross anatomy and light microscopy of placenta, membranes and umbilical cord. An equal size sample of randomized AGA newborns was used. All children were born at Maternidade Terezinha de Jesus, Juiz de Fora - MG, between February and November, 1996. After an informed consent was given by the mothers, the newborns were weighted at birth with assessment of length and head circumference. Every child's placenta, membranes and umbilical cord were sent to the laboratory of histology and embryology of the Department of Morphology of Universidade Federal de Juiz de Fora and Department of Pathology and Forensic Medicine of Universidade Federal de Minas Gerais.
\end{abstract}

Results: placentas of SGA newborns differed significantly with greater incidence of chorioamniotitis, placental infarction, extensive perivillous fibrin deposition and multiple foci of parabasal chronic villitis. They were also lighter and had smaller diameters. Placentary index (placental weight/newborn's weight ratio) was also significantly greater, indicating that although both placenta and small for gestational age newborn presented low weight, placentas impairment was smaller.

Conclusions: placentas of small for gestational age newborns differed significantly if compared to those of adequate gestational age.

JPediatr (Rio J) 2002; 78 (5): 397-402: small for gestational age infant, fetal growth retardation, placenta.

\section{Introdução}

A inter-relação entre a obstetrícia e a pediatria vem se ampliando progressivamente, notando-se um grande interesse da pediatria pelo estágio intra-uterino da vida, visto 
que essa fase da vida é vital para uma existência pós-natal saudável.

A placenta humana é o centro funcional da unidade mãefeto, desempenhando funções respiratória, nutritiva, excretora, endócrina e imunológica. Agressões que incidam sobre a unidade útero-placentária poderão desviar o feto do seu potencial genético de crescimento, determinando crescimento intra-uterino retardado (CIUR).

Há muito tempo, pesquisadores vêm chamando a atenção para o proveito que se pode auferir do exame anatomopatológico da placenta. A literatura apresenta numerosos estudos relacionando recém-nascidos pequenos para a idade gestacional (PIG) e patologia placentária. A associação com placentas de menor peso tem sido descrita na literatu$\mathrm{ra}^{1-3}$. Outros estudos vêm enfatizando a incidência mais elevada de corioamnionite em placentas de recém-nascidos $\mathrm{PIG}^{4,5}$, relacionando-a com fatores socioeconômicos, amniorrexe prolongada e infecção ascendente. A associação entre infarto placentário e retardo do crescimento intrauterino é também amplamente descrita na literatura ${ }^{1,6-11}$, bem como sua associação com deposição de fibrina perivilosa $a^{2,6,9,10,12}$. Um achado anatomopatológico amplamente estudado é a associação encontrada entre vilosite crônica e retardo do crescimento intra-uterino ${ }^{13-15,17,18}$, indicando que processos inflamatórios no território viloso, reduzindo as trocas materno ovulares, poderiam levar a um processo de desnutrição intra-uterina. A presença de lesões isquêmicas correspondentes a distúrbios circulatórios placentários foi destacada em $43 \%$ dos casos de vilosite ${ }^{13}$.

O presente trabalho teve por objetivo avaliar a morfologia placentária de recém-nascidos a termo, pequenos e adequados para a idade gestacional, através de exame macroscópico e de microscopia óptica da placenta, membranas e cordão umbilical.

\section{População, material e método}

Realizou-se estudo transversal, no qual foram coletados dados referentes a recém-nascidos a termo estimados como pequenos para a idade gestacional pela curva de Lubchenco et al. ${ }^{19,20}$. Foram estudadas todas as placentas de crianças PIG nascidas, consecutivamente, na Maternidade Terezinha de Jesus, em Juiz de Fora, MG, no período compreendido entre fevereiro a novembro de 1996, e que não se enquadravam nos critérios de exclusão da pesquisa. Como grupo controle, foram incluídos, aleatoriamente, recémnascidos a termo adequados para a idade gestacional, randomizados a partir do número de ordem de nascimento, na proporção de um controle para cada caso. O tamanho da amostra foi condicionado pelo intervalo de tempo programado para a coleta dos dados e pela limitação imposta pela escassez de material de laboratório utilizado no processamento das placentas.

Foram considerados critérios de exclusão do estudo, uma ou mais das condições abaixo relacionadas:
- afecções neurológicas neonatais que impedissem a avaliação da idade gestacional do recém-nascido pelo método de Capurro, somatoneurológico;

- evolução clínica imediata que inviabilizasse ou interferisse na obtenção das medidas propostas;

- malformações congênitas múltiplas;

- gestação múltipla;

- acretismo placentário ou outra condição placentária que alterasse a integridade da placenta ou das membranas;

- infecção congênita diagnosticada durante o pré-natal.

No total, foram coletados dados de 50 recém-nascidos PIG e o mesmo número de recém-nascidos adequados para a idade gestacional (AIG). Dos 100 recém-nascidos participantes do estudo, foram obtidas as placentas, cordão umbilical e membranas, que foram processadas e examinadas no Laboratório de Histologia e Embriologia do Departamento de Morfologia da UFJF e Departamento de Anatomia Patológica e Medicina Legal da UFMG, baseando-se na técnica descrita por Fox ${ }^{5}$.

As placentas coletadas foram examinadas macroscopicamente, após retirada do excesso de sangue, retirada das membranas e corte do cordão umbilical a 1 centímetro do disco placentário. Após, foram fixadas em formol a $10 \%$ durante um período médio de 8 dias, quando realizavam-se cortes paralelos do disco placentário. Foram retiradas rotineiramente amostras da placenta, do cordão e das membranas para o preparo de cortes histológicos - cordão umbilical: dois cortes transversais em extremidades opostas; membranas: um corte transversal do "rolinho"; placenta: uma amostra de cada quadrante, além de amostras de regiões suspeitas. Os fragmentos selecionados passaram pelo processamento histológico de rotina, submetidos à coloração pela hematoxilina-eosina. A análise histológica dos cortes foi efetuada por meio de microscopia óptica pela orientadora da pesquisa, a qual não tinha conhecimento sobre a classificação dos recém-nascidos. Os achados histopatológicos observados foram fotografados para documentação.

As mães foram entrevistadas, e os recém-nascidos avaliados quanto à idade gestacional, peso, comprimento e perímetro cefálico. Calculou-se também o índice placentário dividindo-se o peso da placenta pelo peso do recémnascido, ambos em gramas. Para a estimativa da idade gestacional, foi utilizada a informação obtida da mãe sobre a data da última menstruação, quando as mães afirmavam com certeza, e confirmada, quando possível, pelo ultra-som realizado antes de 20 semanas de gestação e pelo exame clínico-neurológico proposto por Capurro et al. ${ }^{21}$, respeitando-se uma diferença de até duas semanas.

Uma vez concluídos, os questionários foram revisados, codificados, digitados e analisados pelo software Epi-Info 6.0.

Este estudo foi submetido à apreciação prévia e obteve aprovação e autorização da Diretoria da Maternidade Terezinha de Jesus, dos Departamentos de Morfologia e Mater- 
no-Infantil, ambos da UFJF, do Departamento de Anatomia Patológica e Medicina Legal da UFMG, além das Comissões de Normas Técnicas e Ética ou equivalentes. Na UFMG, foi encaminhado ao COEP - Comitê de ética em pesquisa - para apreciação, sendo igualmente aprovado.

\section{Resultados}

A idade das mães variou de 13 a 44 anos. Em relação à escolaridade, $72 \%$ das mães apresentavam primeiro grau incompleto. Somente $2 \%$ apresentavam o segundo grau completo. As mães pertenciam, em sua maioria, a famílias de baixa renda, sendo $61 \%$ provenientes de famílias com renda mensal menor que um salário mínimo per capita. Quanto à situação conjugal, 27\% das mães viviam separadas do pai da criança por ocasião do parto.

Observou-se que $69 \%$ das puérperas não fizeram uso de nenhum cigarro durante a gravidez, assim como $91 \%$ delas não relataram uso de bebida alcoólica. Em um caso, houve relato do uso de cocaína e maconha.

Dentre os principais diagnósticos ou condições clínicas encontradas, observou-se 7\% de doença hipertensiva específica da gravidez (DHEG), 5\% de hipertensão arterial crônica, e 3\% de anemia grave.

Verificou-se que nove mães não realizaram pré-natal. Dentre as gestantes que realizaram pré-natal, a média de consultas foi de $5,9( \pm 2,1)$. Quanto à paridade, $33 \%$ das mães dos recém-nascidos eram primíparas, e $5 \%$ tinham tido mais de quatro gestações. Considerando apenas as multíparas $(\mathrm{n}=67)$, verificou-se a presença de história anterior positiva para recém-nascido de baixo peso em 19 mães. Houve um leve predomínio do parto do tipo cesareana, com uma incidência de $52 \%$. Entre os recém-nascidos, houve um predomínio do sexo feminino, com incidência de 58,0\%.

A Tabela 1 demonstra alguns dados que revelaram uma favorável tendência de associação com o nascimento de recém-nascidos PIG. Tais dados incluem a idade maternamães menores que 16 anos e maiores que 35 anos $(\mathrm{p}<0,05)$, pré-natal - ausência de pré-natal ou realização de apenas uma consulta $(\mathrm{p}<0,01)$, paridade - primíparas e multíparas $>4(\mathrm{p}<0,05)$, e história de recém-nascido com baixo peso em gestação anterior $(\mathrm{p}<0,05)$.

A média dos pesos dos recém-nascidos de toda a amostra foi de $2.783 \pm 436 \mathrm{~g}$; a mediana foi $2.670 \mathrm{~g}$, o peso mínimo foi $1.920 \mathrm{~g}$ e o máximo, $3.640 \mathrm{~g}$. Verificou-se a presença de baixo peso $(<2.500 \mathrm{~g})$ em $27 \%$ dos recémnascidos. A média dos comprimentos foi de $46,5 \pm 2,2 \mathrm{~cm}$; a mediana foi $46,5 \mathrm{~cm}$, o comprimento mínimo foi $41,2 \mathrm{~cm}$ e o máximo, $50,5 \mathrm{~cm}$. A média dos perímetros cefálicos foi de $34 \pm 1,5 \mathrm{~cm}$; a mediana foi $34,1 \mathrm{~cm}$, o PC mínimo foi $30,5 \mathrm{~cm}$ e o máximo $37 \mathrm{~cm}$ (Tabela 2). A distribuição dos recém-nascidos segundo o peso de nascimento nos grupos PIG e AIG pode ser observada na Tabela 3. A distribuição dos recém-nascidos de acordo com o peso de nascimento em relação ao sexo e à idade gestacional pode ser melhor analisada nas Figuras 1 e 2.
Tabela 1 - Características maternas associadas ao nascimento de recém-nascidos PIG, MTJ - FM/UFJF, 1996

\begin{tabular}{lccc}
\hline Características & PIG & AIG & p \\
\hline $\begin{array}{l}\text { Idade materna } \\
\quad<16 \text { ou }>35 \text { anos } \\
\quad \text { entre } 16 \text { e } 35 \text { anos }\end{array}$ & 9 & 2 & \\
$\begin{array}{l}\text { Pré-natal } \\
\quad \text { Ausente ou somente 1 consulta }\end{array}$ & 91 & 48 & $<0,05$ \\
$\quad$ 2 consultas & 35 & 47 & $<0,01$ \\
$\begin{array}{l}\text { Paridade } \\
\quad \text { Primíparas ou multíparas }>4\end{array}$ & 24 & 14 & \\
$\quad$ Multíparas $\leq 4$ & 26 & 36 & $<0,05$ \\
$\begin{array}{l}\text { Recém-nascido de baixo peso } \\
\text { em gestações anteriores }\end{array}$ & & & \\
$\quad \begin{array}{l}\text { Presente } \\
\quad \text { Ausente }\end{array}$ & 12 & & 7 \\
$\quad 13$ & 27 & $<0,05$ \\
\hline
\end{tabular}

Os dados referentes ao exame placentário são descritos a seguir e sintetizados na Tabela 4. Em 86\% dos casos, não foram encontradas alterações de cordão umbilical. As alterações encontradas foram hemorragia, artéria umbilical única, trombose de vasos umbilicais e funiculite, e não se associaram estatisticamente ao nascimento de recém-nascido PIG. Também observou-se uma grande proporção de casos sem nenhuma alteração no exame das membranas (78\%). A alteração mais freqüente nas membranas foi a corioamnionite, verificada em $17 \%$ dos casos, tendo se associado estatisticamente ao nascimento de recém-nascido PIG $(\mathrm{p}<0,05)$.

A média dos pesos placentários foi de $402 \pm 67,2 \mathrm{~g}$, e a mediana foi $392,5 \mathrm{~g}$. Placentas com menor peso se associaram favoravelmente ao nascimento de recém-nascido PIG $(\mathrm{p}<0,00001)$.

O peso da placenta foi também avaliado em relação ao peso do feto através do índice placentário. A média dos índices foi de $0,15 \pm 0,02$, e a mediana foi 0,14 . Quando se

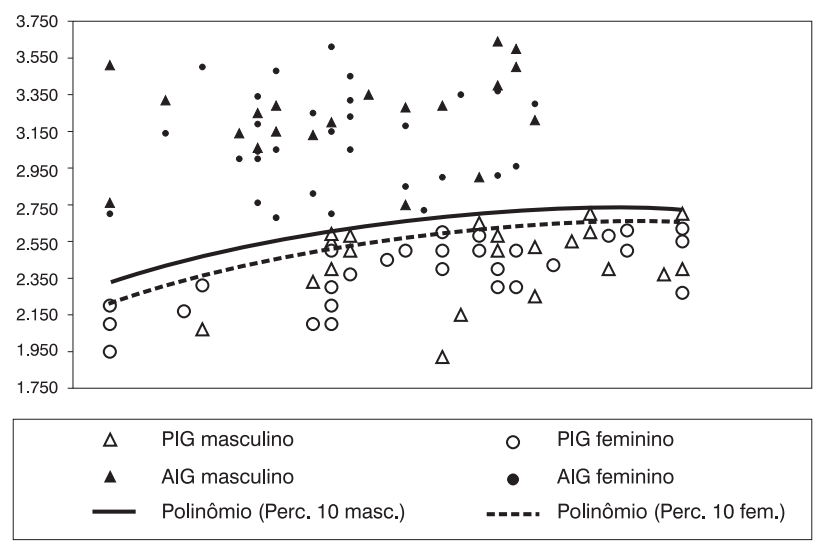

Figura 1 - Distribuição dos recém-nascidos de acordo com o peso de nascimento (em gramas), em relação ao sexo e à idade gestacional (em dias), MTJ - FM/UFJF, 1996 
Tabela 2 - Características antropométricas dos recém-nascidos, MTJ - FM/UFJF, 1996

\begin{tabular}{lccccc}
\hline $\begin{array}{l}\text { Medidas dos } \\
\text { recém-nascidos }\end{array}$ & Média & Mediana & Mínimo & Máximo & Desvio-padrão \\
\hline Total de recém-nascidos & & & & & \\
Peso & $2.783 \mathrm{~g}$ & $2.670 \mathrm{~g}$ & $1.920 \mathrm{~g}$ & $3.640 \mathrm{~g}$ & $436 \mathrm{~g}$ \\
Comprimento & $46,5 \mathrm{~cm}$ & $46,5 \mathrm{~cm}$ & $41,2 \mathrm{~cm}$ & $50,5 \mathrm{~cm}$ & $2,2 \mathrm{~cm}$ \\
Perímetro cefálico & $34,0 \mathrm{~cm}$ & $34,1 \mathrm{~cm}$ & $30,5 \mathrm{~cm}$ & $37,0 \mathrm{~cm}$ & $1,5 \mathrm{~cm}$ \\
Recém-nascidos PIG & & & & & \\
Peso & $2.412 \mathrm{~g}$ & $2.450 \mathrm{~g}$ & $1.920 \mathrm{~g}$ & $2.700 \mathrm{~g}$ & $187 \mathrm{~g}$ \\
Comprimento & $45,1 \mathrm{~cm}$ & $45,4 \mathrm{~cm}$ & $41,2 \mathrm{~cm}$ & $49,4 \mathrm{~cm}$ & $1,9 \mathrm{~cm}$ \\
Perímetro cefálico & $33,0 \mathrm{~cm}$ & $33 \mathrm{~cm}$ & $30,5 \mathrm{~cm}$ & $35,7 \mathrm{~cm}$ & $1,1 \mathrm{~cm}$ \\
\hline
\end{tabular}

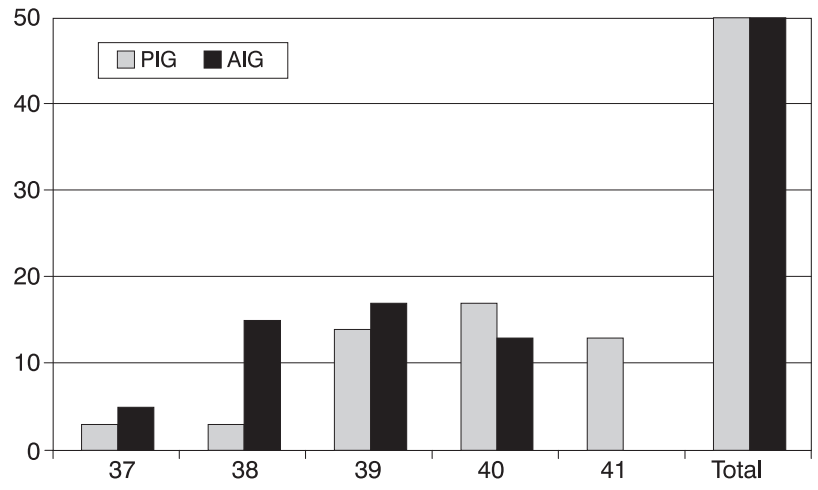

Figura 2 - Distribuição dos recém-nascidos de acordo com a idade gestacional ao nascimento (em semanas completas), MTJ - FM/UFJF, 1986

utilizou a média dos índices placentários, observou-se que índices maiores se associaram estatisticamente ao nascimento de recém-nascidos PIG $(\mathrm{p}<0,05)$.

Observou-se associação significativa entre os diâmetros placentários e a relação peso/idade gestacional dos recém-nascidos, quando se utilizou a média dos diâmetros como ponto de corte. Placentas menores se associaram favoravelmente ao nascimento de recém-nascido PIG $(\mathrm{p}<0,01)$.

Tabela 3 - Distribuição dos recém-nascidos de acordo com o peso de nascimento (em gramas), MTJ - FM/UFJF, 1996

\begin{tabular}{lccc}
\hline Peso de nascimento & $\mathbf{n}$ & PIG & AIG \\
\hline$<2.000$ & 2 & 2 & 0 \\
$2.000-2.249$ & 5 & 5 & 0 \\
$2.250-2.499$ & 20 & 20 & 0 \\
$2.500-2.749$ & 27 & 23 & 4 \\
$2.750-2.999$ & 9 & 0 & 9 \\
$3.000-3.249$ & 17 & 0 & 17 \\
$3.250-3.499$ & 14 & 0 & 14 \\
$\geq 3.500$ & 6 & 0 & 6 \\
\hline Total & 100 & 50 & 50 \\
\hline
\end{tabular}

A presença de pelo menos alguma alteração histopatológica no exame placentário foi verificada em 95\% dos casos. Somente $5 \%$ das placentas examinadas estavam isentas de alterações. Os achados específicos incluíram, com mais freqüência, infartos placentários, deposição de fibrina perivilosa, vilosite crônica, calcificações grosseiras, trombose intervilosa, sinais de imaturidade vilosa, falcização de hemácias maternas no espaço interviloso, alterações vasculares, além de outros achados de ocorrência esporádica.

Observou-se associação estatisticamente significante entre infarto placentário $(\mathrm{p}<0,01)$ e deposição de fibrina perivilosa $(p<0,000001)$, com o nascimento de recémnascido PIG. A presença de pelo menos algum foco de vilosite foi observada em $24 \%$ das placentas. A presença de focos múltiplos de localização parabasal foi encontrada em $15 \%$ das placentas. Verificou-se associação estatisticamente significante entre vilosite crônica e o nascimento de recém-nascido PIG, quando se considerou especificamente o achado equivalente a focos múltiplos de localização parabasal $(p<0,05)$. Verificou-se a presença de calcificações grosseiras em $29 \%$ das placentas examinadas, presença de trombose intervilosa em $17 \%$, sinais de imaturidade vilosa relativa ou discreta em $22 \%$, corioangiose em $10 \%$, e hemácias maternas falciformes no espaço interviloso em $8 \%$, embora estes achados não tenham sido estatisticamente significantes em relação ao nascimentos de PIG $(\mathrm{p}>0,05)$.

Alterações vasculares foram observadas em 16\% das placentas, representadas por vilos avasculares, esclerose de vasos vilositários, endovasculite hemorrágica, trombose de vasos vilositários e da placa basal. Outras alterações encontradas foram deciduite crônica, hematoma retroplacentário, hemorragia recente do estroma viloso, placenta extracorial e quarto vaso no cordão umbilical. Estas observações não apresentaram significado estatístico.

\section{Discussão}

Apesar do crescente interesse científico da neonatologia pela placenta, notamos que há ainda escassa literatura que aborde o tema de forma multidisciplinar. Embora sejam 
Tabela 4 - Alterações significativas encontradas ao exame placentário, MTJ - FM/UFJF, 1996

\begin{tabular}{|c|c|c|c|}
\hline Alterações placentárias & PIG & AIG & $\mathbf{p}$ \\
\hline \multicolumn{4}{|l|}{ Corioamnionite } \\
\hline Presente & 13 & 4 & \\
\hline Ausente & 37 & 45 & $<0,05$ \\
\hline \multicolumn{4}{|l|}{ Peso placentário } \\
\hline$<402 \mathrm{~g}$ & 41 & 17 & \\
\hline$\geq 402 \mathrm{~g}$ & 9 & 33 & $<0,00001$ \\
\hline \multicolumn{4}{|l|}{ Índice placentário } \\
\hline$<0,15$ & 24 & 36 & \\
\hline$\geq 0,15$ & 26 & 14 & $<0,05$ \\
\hline \multicolumn{4}{|l|}{ Diâmetro placentário } \\
\hline$<18,2$ & 34 & 19 & \\
\hline$\geq 18,2$ & 16 & 31 & $<0,01$ \\
\hline \multicolumn{4}{|c|}{ Deposição perivilosa de fibrina } \\
\hline Presente & 28 & 4 & \\
\hline Ausente & 22 & 46 & $<0,000001$ \\
\hline \multicolumn{4}{|l|}{ Vilosite crônica parabasal } \\
\hline Presente & 11 & 4 & \\
\hline Ausente & 39 & 46 & $<0,05$ \\
\hline \multicolumn{4}{|l|}{ Infarto placentário } \\
\hline Presente & 15 & 2 & \\
\hline Ausente & 35 & 48 & $<0,01$ \\
\hline
\end{tabular}

múltiplos os fatores que influenciam o crescimento intrauterino $^{22-25}$, são relativamente poucos os estudos multidisciplinares relacionando alterações de placentas com grupos específicos de crianças pequenas para a idade gestacional. Estudar o envolvimento placentário em nascimentos de crianças PIG é buscar alterações que, direta ou indiretamente, possam associar-se à etiopatogenia do retardo do crescimento intra-uterino.

O achado de placentas de menor peso e menores diâmetros em recém-nascidos PIG estão de acordo com os dados encontrados na literatura ${ }^{1-3}$. Além disso, o índice placentário mostrou-se significativamente maior no grupo PIG. Isso demonstra que, embora ambos - placenta e recém-nascido PIG - tenham apresentado comprometimento do peso, o comprometimento do peso fetal foi relativamente maior que o comprometimento de peso da placenta.

A corioamnionite, que predominou entre os recémnascidos PIG, pode decorrer da penetração de microorganismos provenientes do canal vaginal (infecção ascendente), sendo esta a causa mais comum no ser humano ${ }^{26}$. Está bem estabelecida a associação entre corioamnionite e restrição do crescimento fetal ${ }^{5}$.

A ocorrência simultânea de infarto placentário e retardo do crescimento intra-uterino aponta para a hipóxia intraútero como um denominador comum. Trata-se de necrose de coagulação do tecido viloso, secundária à oclusão de vasos do leito placentário em casos de má adaptação vascular durante o processo de placentação ${ }^{17}$. Estudos realizados com dopplerfluxometria, em casos de retardo do crescimento intra-uterino, vêm demonstrando uma diminuição do fluxo sangüíneo útero-placentário associado a um aumento na resistência vascular como causa de hipóxia crônica e crescimento intra-uterino retardado ${ }^{10,27-29}$. Esse aumento de resistência nos vasos útero-placentários tem sido atribuído a ausência de modificações fisiológicas nas artérias espiraladas miometriais, devido à falha da segunda onda de migração trofoblástica sobre estes vasos e, embora possa ocorrer em outras condições, é freqüentemente associado à doença hipertensiva específica da gestação. A presença de infartos, apesar de não ser primariamente a causa do retardo do crescimento fetal, contribui para o seu agravamento, ao restringir a superfície vilositária de trocas.

Deposição extensa de fibrina perivilosa tem sido relacionada à diminuição da circulação no espaço interviloso e, freqüentemente, associada à presença de infarto placentário ${ }^{12}$. Tanto o infarto placentário quanto a deposição de fibrina perivilosa têm como causas comuns a redução do fluxo sangüíneo útero-placentário e a hipóxia. Da mesma forma que o infarto placentário, a deposição de fibrina perivilosa causa restrição da superfície vilositária de trocas materno-ovulares e, embora possa não ser a causa primária do crescimento intra-uterino retardado, secundariamente pode agravá-lo.

Vilosite crônica é um importante achado patológico na placenta, em sua maioria de etiologia desconhecida. Sua incidência, segundo dados da literatura, varia de $6 \%$ a $26 \%{ }^{15}$. Sendo um processo inflamatório no território viloso, leva a um processo de desnutrição intra-uterina através da redução das trocas materno-fetais. Vilosite pode ser encontrada em casos de infecções específicas, como citomegalovírus, toxoplasmose, rubéola, sendo que estes casos de etiologia infecciosa específica perfazem uma incidência que varia de três a $8,7 \%$ entre todos os casos de vilosite ${ }^{13,16,20}$. A vilosite pode ser considerada como uma expressão morfológica da resposta fetal a um estímulo antigênico externo, podendo resultar de uma infecção materna ou de uma resposta materna de natureza imune contra os tecidos placentários ${ }^{30}$. Segundo os mesmos autores, na ausência da identificação de infecções transplacentárias, a vilosite deve ser considerada como de etiologia desconhecida, a qual é citada na literatura como a forma mais freqüente de vilosite crônica e fortemente associada ao retardo de crescimento intra-uterino. Vários autores, estudando vilosite crônica idiopática, não encontraram nenhuma variação significativa quanto à idade materna, nível socioeconômico, nutrição, estado conjugal, tabagismo, uso de drogas ou episódios de infecção aguda durante a gravidez $z^{15,18}$. Há indícios de forte associação entre vilosite crônica idiopática e retardo do crescimento intra-uterino em casos de retardo do crescimento intra-uterino recorrente - diagnosticado em duas gestações a termo consecutivas sem nenhuma evidência de infecção nas mães ou nos recémnascidos ${ }^{17}$. Foi estabelecida associação entre o grau de vilosite e retardo de crescimento intra-uterino, encontrando maior incidência de retardo do crescimento intra-uterino entre os quadros de vilosite crônica mais grave ${ }^{18}$. Em nosso 
estudo, a associação foi significativa quando considerados os quadros de vilosite crônica de focos múltiplos e localização parabasal.

Os achados de nossa pesquisa coincidem com aqueles apontados pela literatura internacional, relevantes na associação com o retardo do crescimento intra-uterino e o nascimento de recém-nascidos PIG. Acreditamos que a patologia placentária muito pode contribuir para a compreensão do complexo tema do retardo do crescimento intrauterino. Seria interessante o desenvolvimento de estudos interdisciplinares longitudinais envolvendo as áreas de obstetrícia, pediatria e anatomia patológica, que pudessem acompanhar a gestante e o crescimento fetal desde as primeiras consultas de pré-natal até o nascimento, com posterior acompanhamento pediátrico da evolução clínica e do crescimento e desenvolvimento do recém-nascido até os primeiros meses de vida. A avaliação anatomopatológica placentária poderia ser mais abrangente, utilizando-se outras técnicas além da rotina, tais como a imunohistoquímica, métodos morfométricos mais detalhados, além da análise microbiológica e parasitológica. Poder-se-ia estudar um número de casos maior, através de uma amostra representativa de uma determinada população, e pesquisar todas as variáveis sabidamente envolvidas no retardo do crescimento intra-uterino, o que idealmente permitisse uma avaliação epidemiológica da pesquisa.

\section{Agradecimentos}

À Maternidade Terezinha de Jesus, onde o trabalho de campo e a coleta de dados foram desenvolvidos; ao Departamento de Morfologia da UFJF, setor de técnica histológica, onde todo o material coletado foi processado; e, em particular, ao técnico de histologia Sebastião Teodoro, pela sua dedicação e desprendimento no preparo dos cortes histológicos.

\section{Referências bibliográficas}

1. Bjoro K Jr. Gross pathology of the placenta in intrauterine growth retardation. Ann Chir Gynaecol 1981;70:316-22.

2. Paiva de Alvarez S, Guevara J, Guevara B. Analisis de la placenta en el retardo del crecimiento intrauterino. Obstet Ginecol Latinoam 1986;44:179-86.

3. Darbon Grosso HA, Tarragona MS, Cristaldi MR. Estudio anatomopatologico en el retardo del crecimiento intrauterino idiopático. Obstet Ginecol Latinoam 1986;44:132-4.

4. Fox H. Pathology of the placenta. Philadelphia: WB Saunders; 1978.

5. Williams MC, O’Brien WF, Nelson RN, Spellacy WN. Corioamnionite histológica está associada a restrição do crescimento fetal em bebês de termo e pré-termo. Am J Obstet Gynecol 2000;183:1094-9.

6. Grundmann E. Perinatal pathology. Curr Top Pathol 1979;66:2-55.

7. Sandstedt B. The placenta and low birth weight. Curr Top Pathol 1979;66:1-56.

8. Trindade CEP. Alterações anatomopatológicas placentárias: relação com o peso e idade gestacional de recém-nascidos. J Pediatr (Rio J) 1980;48:146-51.

9. De Len Prera R, Herrera Ortiz R, Rivera C. Influencia de patologia placentária en el recin nascido pequeño para edad gestacional. Guatem Pediatr 1986;8:239-47.
10. Salafia CM, Vintzileos AM, Silberman L. Placental pathology of idiopathic intrauterine growth retardation at term. Am J Perinatol 1992;9:179-84.

11. Becroft DM, Thompson JMD, Mitchell EA. The epidemiology of placental infarction at term. Placenta 2002;23:343-51.

12. Shanklin DR. The influence of placental lesions on the newborn infant. Pediatr Clin North Am 1970;17(1):25-42.

13. Altshuler G, Russel P, Ermocilla R. The placental pathology of small for gestational age infants. Am J Obstet Gynecol 1975; 121:351-9.

14. Russel P. Inflammatory lesions of the human placenta. The histopathology of villitis of unknown aetiology. Placenta 1980;1:227-44.

15. Knox WF, Fox H. Villitis of unknown aetiology: its incidence and significance in placentae from a British population. Placenta 1984;5:395-402.

16. Ramos HIB. A placenta do recém-nascido baixo peso. In: Garcia A, Azoubel RA. Placenta humana: morfologia e patologia fetal e perinatal. Rio de Janeiro: Atheneu; 1986.

17. Labarrere $\mathrm{C}$, Althabe $\mathrm{O}$. Chronic villitis of unknown aetiology in recurrent intrauterine fetal growth retardation. Placenta 1987;8:167-73.

18. Nordenvall M, Sandstedt B. Placental villitis and intrauterine growth retardation in a Swedish population. APMIS 1990; 98:19-24.

19. Lubchenco LO, Hansman C, Dressler M. Intrauterine growth as estimated from liveborn birth-weight data at 24 to 42 weeks of gestation. Pediatrics 1963;32:791-800.

20. Lubchenco LO, Hansman C, Boyd E. Intrauterine growth in length and head circumference as estimated from live births at gestational ages from 26-42 weeks. Pediatrics 1966;37:403-08.

21. Capurro H, Konichezky S, Fonseca D. A simplified method for diagnosis of gestational age in the newborn infant. J Pediatr 1978;93:120-2.

22. Yunes J. Principais fatores maternos associados à ocorrência de recém-nascidos de baixo peso. J Pediatr (Rio J) 1978;44:279-83.

23. Matsubara S, Sato I. Placenta of idiopathic fetal growth restriction. Placenta 2000;21:257-62.

24. Luckas MJM, Sandland R, Hawe J, Neilson JP, McFadyen IR, Meekins JW. Fetal growth retardation and second trimester maternal serum human chorionic gonadotrophin levels. Placenta 1998;19(2/ 3):143-7.

25. Sikkema JM, Franx A, Bruinse HW, van der Wijk NG, de Valk HW, Nikkels PGJ. Placental pathology in early onset pre-eclampsia and intra-uterine growth restriction in women with and without thrombophilia. Placenta 2002;23:337-42.

26. Driscoll SG. Antenatal infection and unruptured membranes. Pediatrics 1970;45:886-9.

27. Karsdorp VHM, Dirks BK, van der Linden JC, van Vugt JMG, Baak JPA, van Geijn HP. Placenta morphology and absent or reversed end diastolic flow velocitites in the umbilical artery: a clinical and morphometrical study. Placenta 1996;17:393-9.

28. Ferrazzi E, Bulfamante G, Mezzopane R, Barbera A, Ghidini A, Pardi G. Uterine doppler velocimetry and placental hypoxic-ischemic lesion in pregnancies with fetal intrauterine growth restriction. Placenta 1999;20:389-94.

29. Pardi G, Marconi AM, Cetin I. Placental-fetal interrelationship in IUGR fetuses. Placenta 2002;23:136-41.

30. Benirschke K, Kaufmann P. Infections diseases. In: Benirschke K, Kaufmann P. Pathology of the human placenta. $3^{\mathrm{a}}$ ed. New York: Springer-Verlag; 1995.

Endereço para correspondência

Dr. Lúcio Henrique de Oliveira

Faculdade de Medicina - Univ. Federal de Juiz de Fora

Rua Vicente Adão Botti, 65/301 - Bom Pastor

CEP 36021-550 - Juiz de Fora, MG

Fone: (32) 3218.8055 - E-mail: lucio@icb.ufjf.br 